\title{
Fenofibrato - un agonista de PPAR $\alpha$ - incrementa los niveles de la alcohol deshidrogenasa hepática: implicaciones para su posible uso como una droga de aversión al etanol
}

\author{
Fenofibrate -a PPAR o agonist- increases alcohol \\ dehydrogenase levels in the liver: implications for \\ its possible use as an ethanol-aversive drug
}

\author{
Daniel Muñoz*, Mario Rivera-Meza***, Osvaldo Flores-Bastías*, **, \\ María Elena Quintanilla****, Eduardo Karahanian*, **. \\ * Instituto de Ciencias Biomédicas, Facultad de Ciencias de la Salud. ** Centro de Investigación para el Estudio de la Conducta \\ del Consumo de Alcohol en Adolescentes. Universidad Autónoma de Chile, Santiago. Chile. *** Departamento de Química \\ Farmacológica y Toxicológica, Facultad de Ciencias Químicas y Farmacéuticas. Universidad de Chile, Santiago. Chile. \\ **** Programa de Farmacología Molecular y Clínica, Facultad de Medicina, Instituto de Ciencias Biomédicas. Universidad de \\ Chile, Santiago. Chile.
}

\section{Resumen}

Tras consumir etanol, el disulfiram incrementa los niveles de acetaldehído en sangre y genera una reacción aversiva que desalienta el consumo de alcohol. Dados los importantes efectos secundarios del disulfiram, es altamente deseable hallar otros fármacos efectivos para tratar el trastorno por uso de alcohol. Se ha reportado que administrar fenofibrato a ratas altamente bebedoras de alcohol aumenta los niveles de catalasa hepática y acetaldehído en sangre después de la administración de etanol, y disminuye el consumo voluntario de alcohol (60-70\%). Este trabajo evalúa si el fenofibrato tiene un efecto adicional sobre la actividad de otras enzimas en el metabolismo del etanol que podría contribuir a generar altos niveles de acetaldehído. Se permitió a ratas macho altamente bebedoras beber voluntariamente etanol $10 \%$ durante 2 meses. Después, se les administró oralmente fenofibrato (100 mg/kg/ día) o solo vehículo durante 14 días. Tras eso, se midieron los niveles hepáticos y actividades enzimáticas de alcohol deshidrogenasa (ADH1) y de aldehído deshidrogenasa (ALDH2). El fenofibrato produjo un marcado aumento en los niveles proteicos de ADH1 (396\% $\pm 18 \%, \mathrm{p}<$ $, 001)$ y de actividad enzimática $(425 \% \pm 25 \%, \mathrm{p}<, 001)$ sin alterar los niveles protéicos ni la actividad de ALDH2. Los resultados muestran que el tratamiento con fenofibrato no solo aumenta la actividad de catalasa en el hígado de ratas bebedoras de alcohol, sino que también incrementa los niveles y la actividad de ADH1, sin alterar ALDH2. Esto contribuye a explicar el notable efecto del fenofibrato en aumentar los niveles de acetaldehído en sangre en animales bebedores de alcohol, en los que se registra una marcada reducción en la ingesta de etanol.

Palabras clave: Fibrato; Receptor activado por proliferadores de peroxisomas; PPAR; Alcohol deshidrogenasa; Tratamiento trastorno por uso de alcohol.

\section{Abstract}

After ethanol consumption, disulfiram increases blood-acetaldehyde levels, generating an aversive reaction that deters alcohol drinking. Given the major secondary effects of disulfiram, finding other effective drugs to reduce alcohol consumption in individuals with alcohol-use-disorder is highly desirable. It has been reported that administering fenofibrate to high-drinking rats increases hepatic catalase levels and blood acetaldehyde after administering ethanol and a $60-70 \%$ inhibition of voluntary alcohol intake. This work evaluated whether fenofibrate has an additional effect on the activity of other ethanol-metabolizing enzymes, which could contribute to the high acetaldehyde levels generated upon administering ethanol. Male highdrinker rats were allowed to voluntary drink $10 \%$ ethanol or water for 2 months. Subsequently, fenofibrate $(100 \mathrm{mg} / \mathrm{kg} /$ day $)$ or vehicle was administered orally for 14 days. Then, alcohol dehydrogenase (ADH1) and aldehyde dehydrogenase (ALDH2) protein levels and enzymatic activities in the livers were quantified. Fenofibrate treatment produced a marked increase in ADH1 protein levels $(396 \% \pm 18 \%, \mathrm{p}$ $<0.001)$ and enzymatic activity $(425 \% \pm 25 \%, \mathrm{p}<0.001)$. Fenofibrate did not result in differences in ALDH2 activity or in ALDH2 protein levels. The studies show that treatment with fenofibrate not only increased the activity of catalase in the liver of alcohol-drinking rats, as reported earlier, but also increased the levels and enzymatic activity of ADH1, while ALDH2 remained unchanged. The increases in ADH1 contribute to explaining the remarkable effect of fenofibrate in raising blood levels of acetaldehyde in ethanol-consuming animals, in which a marked reduction of alcohol intake is recorded.

Key Words: Fibrate; Peroxisome proliferator-activated receptor; PPAR; Alcohol dehydrogenase; Alcohol use disorder treatment.

Recibido: Agosto 2018; Aceptado: Noviembre 2018.

Enviar correspondencia a: Dr. Eduardo Karahanian. Instituto de ciencias biomédicas, Facultad de ciencias de la salud - Universidad Autónoma de Chile. Llano Subercaseaux 2801, $5^{\circ}$ piso. San Miguel 8910060, Santiago - Chile. Tel: 56-223036664.

E-mail: eduardo.karahanian@uautonoma.cl. 
$\mathrm{E}$ xisten tres medicamentos aprobados para tratar el trastorno por uso de alcohol: naltrexona, acamprosato y disulfiram. A pesar de su uso a escala mundial, la eficacia de estas drogas es bastante limitada (entre 10-13\%) (Maisel, Blodgett, Wilbourne, Humphreys y Finney, 2013; Skinner, Lahmek, Pham y Aubin, 2014). El disulfiram inhibe la aldehído deshidrogenasa (ALDH2) y, por tanto, aumenta los niveles de acetaldehído en sangre en consumidores de alcohol. La rápida acumulación de acetaldehído tiene efectos disfóricos que disuaden un consumo adicional del alcohol. No obstante, el disulfiram tiene efectos secundarios importantes que limitan, de manera significativa, su uso clínico (Dupuy et al., 1995; Mark et al., 2003). Es más, el disulfiram carece de efectividad para reducir los niveles de consumo de alcohol en un porcentaje considerable de pacientes (Christensen, Moller, Ronsted, Angelo y Johansson, 1991). Un ensayo clínico reciente mostró que el disulfiram no es significativamente diferente del placebo en promover la abstinencia (Yoshimura et al., 2014).

En un intento de hallar fármacos mejores para sustituir al disulfiram en la práctica clínica, hemos evaluado el efecto de fenofibrato. Fenofibrato pertenece a la familia de moléculas sintéticas llamadas fibratos (que incluyen clofibrato, gemfibrozilo, ciprofibrato, bezafibrato y fenofibrato), agonistas del receptor activado por proliferadores de peroxisomas (PPAR $\alpha$ ) (Gervois, Fruchart y Staels, 2007). La activación de PPAR $\alpha$ en el hígado aumenta la actividad peroxisomal en este órgano, lo que causa una tasa elevada de oxidación de los ácidos grasos. En base a este efecto, los fibratos tienen un uso clínico amplio para tratar los trastornos con altos niveles de triglicéridos en sangre (Gervois et al., 2007). Otra consecuencia de esta actividad peroxisomal elevada es el aumento de los niveles hepáticos de la catalasa (Karahanian, Quintanilla, Fernandez y Israel, 2014; Rivera-Meza et al., 2017). Como ocurre con la alcohol deshidrogenasa (ADH1) y el citocromo P450 2E1 (CYP2E1), la catalasa es capaz de oxidar el etanol para convertirlo en acetaldehído (Handler y Thurman, 1988a, 1988b). En estudios anteriores, mostramos que la administración de fenofibrato a ratas UChB (Universidad de Chile Bibulous) altamente bebedoras de alcohol produjo un aumento marcado (2,5 veces) de los niveles hepáticos de la catalasa, además de un aumento de 10 veces (hasta $95 \mu \mathrm{M})$ de los niveles de acetaldehído en sangre cuando se administró $1 \mathrm{~g} / \mathrm{kg}$ de etanol a estos animales (Karahanian et al., 2014; Rivera-Meza et al., 2017). Como consecuencia, se observó una reducción voluntaria del consumo de alcohol de 60-70\% cuando se midió en 24 horas, pero la reducción aumentó a 85-90\% cuando el consumo se midió en las primeras 2 horas del ciclo de oscuridad (cuando los animales beben más proporcionalmente). De manera similar, Blednov, Black, Benavidez, Stamatakis y Harris (2016) y Haile y Kosten (2017) informaron que el fenofibrato atenuó la autoadministración de alcohol en ratones y ratas, respectivamente. Además, este efecto fue completamente dependiente de PPAR $\alpha$, dado que la administración de un antagonista de PPAR $\alpha$, o el uso de ratones knockout sin este receptor, no mostraron efecto alguno (Blednov et al., 2016)

Dados los resultados prometedores anteriores, nuestro estudio analizó si el aumento de la actividad de la catalasa era la única responsable del efecto sorprendente del fenofibrato en aumentar los niveles de acetaldehído en sangre, o si también hubieron cambios en los niveles proteicos de ADH1 o ALDH2 y/o en las actividades enzimáticas en los hígados de estos mismo animales. Para este fin, medimos la actividad hepática de ADH1 y ALDH2 y cuantificamos los niveles protéicos mediante Western blot.

\section{Métodos}

\section{Animales}

En este estudio se usaron ratas UChB altamente bebedoras, derivadas de la cepa Wistar y criadas de manera selectiva por su ingesta alta de alcohol (Quintanilla, Israel, Sapag y Tampier, 2006). Ratas macho con una edad de dos meses fueron colocadas en jaulas individuales en ambiente con temperatura y humedad controladas, sujetas a un ciclo regular de 12 horas luz/12 horas oscuridad. Durante 60 días, las ratas podían elegir entre una solución de etanol $10 \%(\mathrm{v} / \mathrm{v})$ y agua de dos tubos graduados (únicamente agua para el grupo de control). La comida (formula para ratas Mardones, Alimentos Cisternas, Santiago, Chile) estaba disponible ad libitum y se registró el volumen de consumo diario de agua y etanol. Después de este periodo, el consumo de etanol se estabilizó en $\sim 7 \mathrm{~g}$ etanol $/ \mathrm{kg} /$ día. El Comité de Bioética sobre Investigación en Animales de la Facultad de Medicina de la Universidad de Chile revisó y aprobó todos los procedimientos aplicados en este estudio (Protocolo CBA0767FMUCH).

\section{Tratamiento con fenofibrato}

El tratamiento con fenofibrato ya fue descrito en detalle y los datos reportados aquí fueron obtenidos de los hígados (congelados a una temperatura de $-80^{\circ} \mathrm{C}$ ) de los animales objeto de dicho informe (Rivera-Meza et al., 2017). Brevemente, tras 60 días de libre elección continuo (24 h/ día) entre una solución de etanol $10 \%$ (v/v) y agua (únicamente agua para el grupo de control), la ingesta de etanol se estabilizó en $\sim 7 \mathrm{~g} / \mathrm{kg} /$ día. Después, se repartieron a los animales en 4 grupos $(\mathrm{n}=6$ ratas por grupo): un grupo de etanol y un grupo de control (agua) recibieron tratamiento con fenofibrato micronizado (Fibronil, Royal Pharma, Chile) administrado vía oral como suspensión acuosa (10 mL/kg) en una dosis de $100 \mathrm{mg} / \mathrm{kg} /$ día durante 14 días consecutivos. Los otros grupos de etanol y de control recibieron tratamiento con un vehículo (agua, $10 \mathrm{~mL} / \mathrm{kg}$, 
oral) durante 14 días consecutivos (Figura 1). Etanol 10\% (en su caso), agua y comida fueron accesibles ad libitum de manera continua $24 \mathrm{~h}$ /día. Se registró la ingesta diaria de etanol y agua.

\section{Cuantificación de los niveles hepáticos de ADH1 y ALDH2 mediante Western blot y actividad enzimática}

Tras el tratamiento con fenofibrato, se suprimió el etanol durante 24 horas y los animales fueron sacrificados. Se

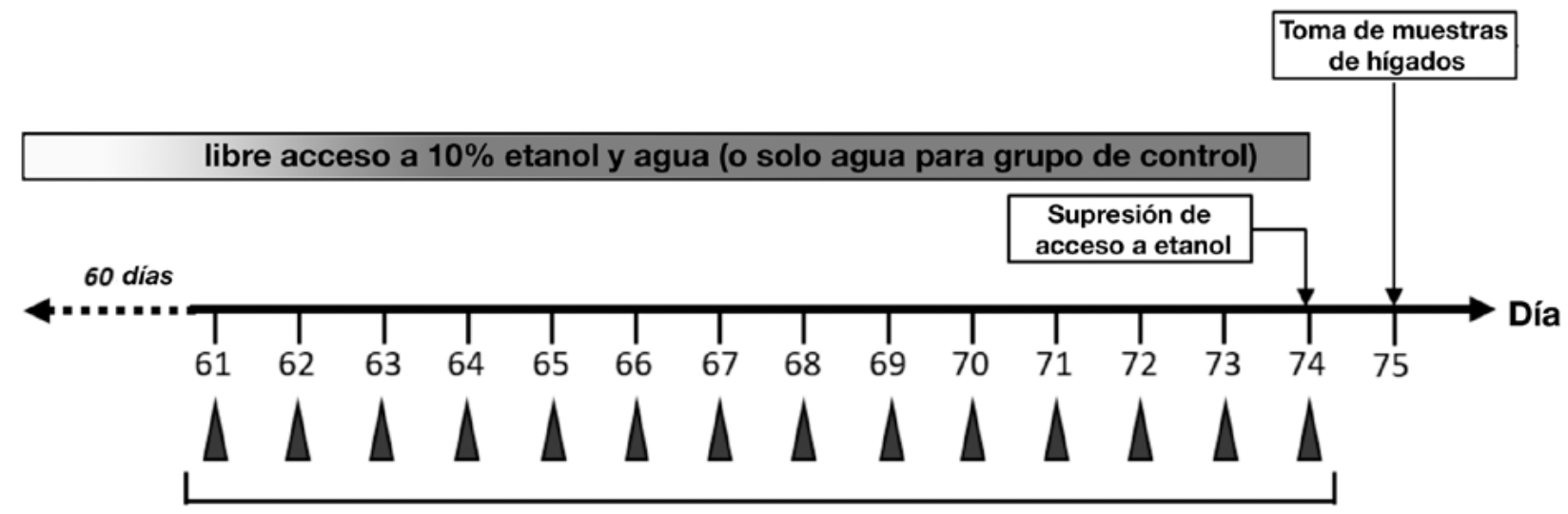

Fenofibrato $(100 \mathrm{mg} / \mathrm{kg})$ o vehículo

Figura 1. Gráfico del calendario temporal para los experimentos de consumo de etanol y de tratamiento con fenofibrato. Doce ratas macho UChB tenían acceso libre las 24 horas entre $10 \% \mathrm{v} / \mathrm{v}$ etanol y agua, y otras doce ratas tenían solo agua (grupo de control) durante 60 días. En el día 61, cada grupo fue dividido en dos subgrupos $(\mathrm{n}=6)$ y cada subgrupo fue administrado, vía oral, $100 \mathrm{mg} / \mathrm{kg} / \mathrm{día}$ de fenofibrato o vehículo, respectivamente, durante 14 días. Tras el tratamiento con fenofibrato, se suprimió el acceso a etanol durante 24 horas, se sacrificaron los animales, y se recolectaron los tejidos hepáticos.

recolectó el tejido hepático y se homogeneizó en un mortero con $1 \%$ Triton X-100 en tampón fosfato [50 mM (pH $7,4)$ ] que contenía un cóctel de inhibidor de proteasas, libre de EDTA (Pierce, Rockford, IL, EE.UU.). Los residuos celulares fueron eliminados mediante centrifugado y el contenido protéico fue determinado con un kit de ensayo de proteínas Micro BCA (Pierce, Rockford, IL, EE.UU.). Las muestras se analizaron mediante el Western blot con anticuerpos primarios de ADH1 (Novus Biologicals NBP212550) y ALDH2 (Novus Biologicals NBP1-52051). Como control de carga, se usó el anticuerpo primario Cell Signaling 8H10D10 para determinar los niveles de b-actina. Los anticuerpos secundarios correspondientes fueron conjugados con HRP. Las membranas fueron reveladas por quimioluminescencia con Pierce ECL Western Blotting Substrate. Las bandas fueron cuantificadas por densitometría con Image J software.

\section{Determinación de las actividades hepáticas de alcohol deshidrogenasa y aldehído deshidrogenasa}

Determinamos las actividades de ADH1 y ALDH2 mediante espectrofotometría en homogeneizados de hígado midiendo la absorción $(340 \mathrm{~nm})$ de nicotinamida-adenina-dinucleótido reducido $(\mathrm{NADH})$ generados a partir de $\mathrm{NAD}+$ en el tiempo. La actividad ADH1 fue medida como se informó anteriormente. (Rivera-Meza et al., 2010): el ensayo se hizo en tampón 0,5 M Tris-HCl ( $\mathrm{pH} 8,0)$ que contenía 0,33 $\mathrm{mM}$ dithiothreitol, $24 \mathrm{mM}$ semicarbazida, $5 \mathrm{mM}$ $\mathrm{NAD}+$, y $100 \mathrm{mg}$ de proteína total. La reacción fue inicia- da al añadir $10 \mathrm{mM}$ etanol. Para la actividad de ALDH2, la mezcla reactiva contenía $40 \mathrm{mM}(\mathrm{pH} 7,4)$ tampón fosfato, $4 \mathrm{mM}$ ditiotreitol, $5 \mathrm{mM} \mathrm{MgCl2,} 10 \mathrm{mM}$ 4-metil-pirazol, 0,8 mM NAD+, y $100 \mathrm{mg}$ de proteína total. La reacción fue iniciada al añadir $14 \mathrm{mM}$ propionaldehído (Karahanian, Ocaranza y Israel, 2005). La actividad específica de ambas enzimas fue expresada como nmol de $\mathrm{NADH} / \mathrm{min} / \mathrm{mg}$ de proteína a $25^{\circ} \mathrm{C}$.

\section{Análisis estadístico}

Analizamos todos los datos mediante análisis de varianza (ANOVA) unidireccional y la prueba Newman-Keuls post hoc.

\section{Resultados}

Como muestra Figura 2, el tratamiento con fenofibrato (F) causó un aumento marcado en los niveles de ADH1 expresados en el análisis Western Blot, tanto en las ratas que consumieron etanol $(\mathrm{E}+\mathrm{F})$ como en las ratas de control que consumieron agua $(\mathrm{A}+\mathrm{F})$, comparado con los animales que no fueron tratados con fenofibrato que solo recibieron agua (A). El aumento fue mucho mayor en el grupo E + F que en el grupo A + F (396\% $\pm 18 \%$ vs $254 \% \pm$ $19 \%, \mathrm{p}<, 001)$. En los animales tratados con etanol (E) que no fueron tratados con fenofibrato (agua como vehículo), hubo un aumento en la expresión de ADH1, comparado con los que solo bebieron agua (A) $(162 \% \pm 9 \%$ vs $100 \%$ $\pm 10 \%, \mathrm{p}<, 05)$. Estos resultados destacan el efecto de etanol solo en el aumento de los niveles hepáticos de ADH1. 

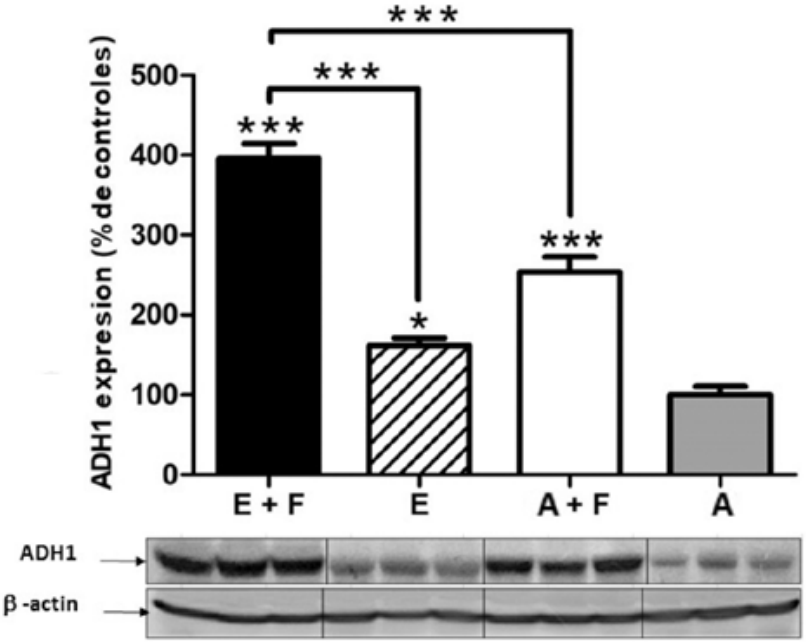

Figura 2. Efecto de fenofibrato en los niveles hepáticos de alcohol deshidrogenasa ( $\mathrm{ADH}_{1}$ ). Las ratas $\mathrm{UChB}$ que consumieron etanol (o agua, para los controles) durante 74 días fueron tratados con fenofibrato $100 \mathrm{mg} / \mathrm{kg} /$ día durante los últimos 14 días de consumo. Los grupos de control respectivos no fueron tratados con fenofibrato (recibieron agua como vehículo). Medimos los niveles de ADH1 mediante Western blot y los normalizamos en relación a los niveles de b-actina. $E+F$ : etanol más fenofibrato; $E$ : etanol; $A$ + F: agua más fenofibrato; $A$ : agua solo, $n=6$ por grupo. El grupo A fue fijado como $100 \%$. Analizamos todos los datos mediante análisis de varianza (ANOVA) unidireccional y la prueba NewmanKeuls post hoc. * representa $p<, 05 y{ }^{* * *}$ representa $p<, 001$. Las barras de error corresponden a SEM. Mostramos los carriles representativos de 3 muestras de cada grupo debajo del gráfico.

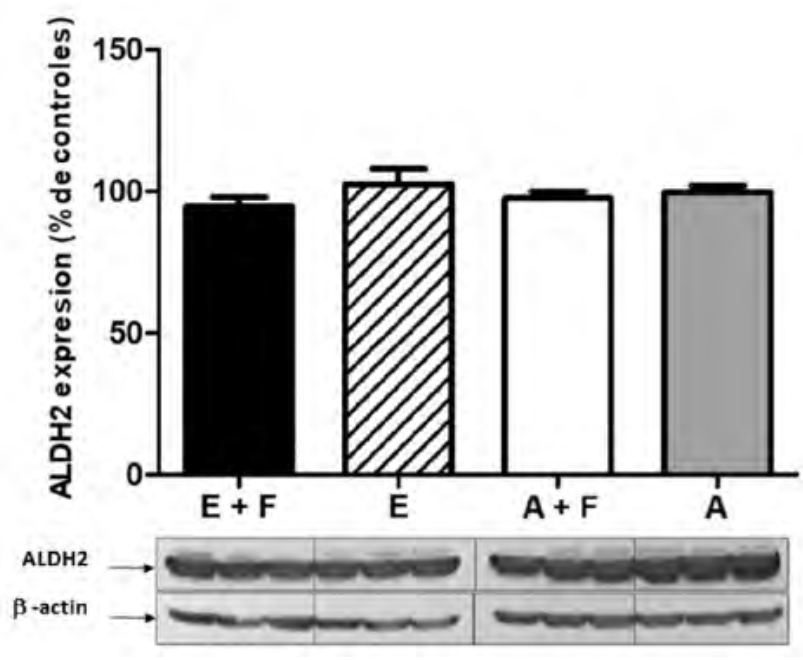

Figura 3. Efecto de fenofibrato en los niveles hepáticos de aldehído deshidrogenasa $(\mathrm{ALDH} 2)$. Las ratas $\mathrm{UChB}$ que consumieron etanol (o agua, para los controles) durante 74 días fueron tratados con fenofibrato $100 \mathrm{mg} / \mathrm{kg} /$ día durante los últimos 14 días de consumo. Los grupos de control respectivos no fueron tratados con fenofibrato (recibieron agua como vehículo). Medimos los niveles de ALDH2 mediante Western blot y los normalizamos en relación a los niveles de b-actina. $E+F$ : etanol más fenofibrato; $E$ : etanol; $A+F$ : agua más fenofibrato; $A$ : agua solo, $n=6$ por grupo. El grupo A fue fijado como $100 \%$. Analizamos todos los datos mediante análisis de varianza (ANOVA) unidireccional y la prueba Newman-Keuls post hoc; no hallamos diferencias significativas. Las barras de error corresponden a SEM. Mostramos los carriles representativos de 3 muestras de cada grupo debajo del gráfico.

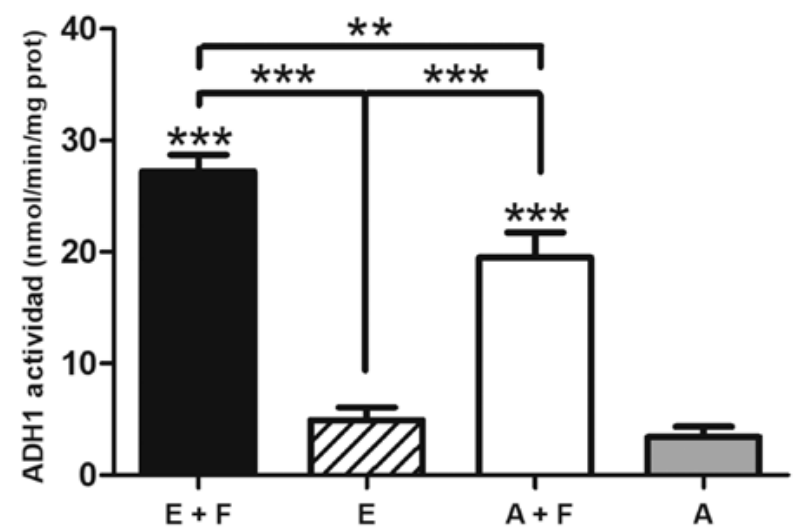

Figura 4. Efecto de fenofibrato en la actividad hepática de alcohol deshidrogenasa $\left(A D H_{1}\right)$. Las ratas UChB que consumieron etanol (o agua, para los controles) durante 74 días fueron tratados con fenofibrato $100 \mathrm{mg} / \mathrm{kg} /$ día durante los últimos 14 días de consumo. Los grupos de control respectivos no fueron tratados con fenofibrato. Actividad $\mathrm{ADH}_{1}$ representa nmol de $\mathrm{NADH} /$ $\mathrm{min} / \mathrm{mg}$ de proteína a $25^{\circ} \mathrm{C}$. E + F: etanol más fenofibrato; E: etanol; $A+F$ : agua más fenofibrato; $A$ : agua solo, $n=6$ por grupo. Analizamos todos los datos mediante análisis de varianza (ANOVA) unidireccional y la prueba Newman-Keuls post hoc.

Nota. ${ }^{* *}$ representa $\mathrm{p}<, 01 \mathrm{y}{ }^{* * *}$ representa $\mathrm{p}<, 001$

Las barras de error corresponden a SEM.

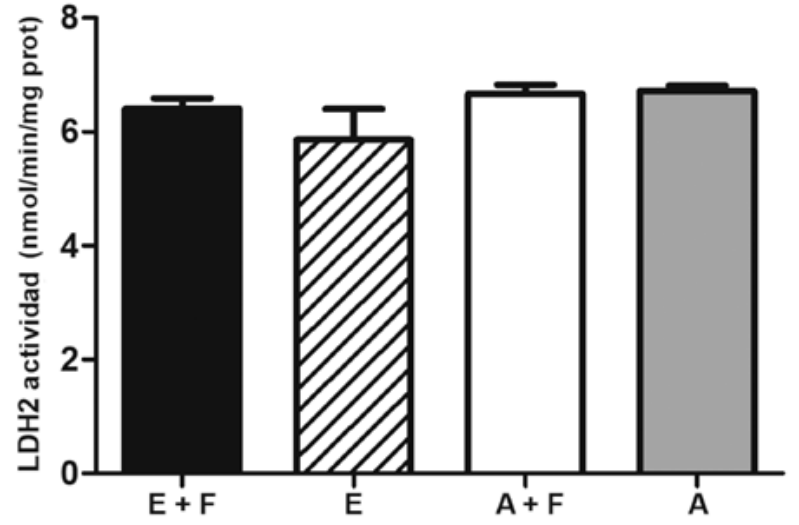

Figura 5. Efecto de fenofibrato en la actividad hepática de aldehído deshidrogenasa $(A L D H 2)$. Las ratas UChB que consumieron etanol (o agua, para los controles) durante 74 días fueron tratados con fenofibrato $100 \mathrm{mg} / \mathrm{kg} /$ día durante los últimos 14 días de consumo. Los grupos de control respectivos no fueron tratados con fenofibrato. Actividad ALDH2 representa nmol de $\mathrm{NADH} /$ $\mathrm{min} / \mathrm{mg}$ de proteína a $25^{\circ} \mathrm{C}$. E + F: etanol más fenofibrato; $\mathrm{E}$ : etanol; $A+F$ : agua más fenofibrato; $A$ : agua solo, $n=6$ por grupo. Analizamos todos los datos mediante análisis de varianza (ANOVA) unidireccional y la prueba Newman-Keuls post hoc. Las barras de error corresponden a SEM. No hallamos diferencias estadísticamente significativas. 
De manera opuesta, como muestra Figura 3, el tratamiento con fenofibrato no tuvo efecto alguno sobre los niveles de proteína ALDH2, ni tampoco hubo un efecto del consumo de alcohol sobre los niveles de esta enzima.

Los resultados de los niveles de proteínas ADH1 y ALDH2 fueron coherentes con la medición de las actividades enzimáticas. Como muestra la Figura 4, el tratamiento con fenofibrato produjo aumentos tanto en la actividad de ADH1 en las ratas que consumieron alcohol, como en las ratas que bebieron solo agua (425\% y $475 \%$, respectivamente, comparado con los controles). En cuanto a la actividad de ALDH2, ni el tratamiento con fenofibrato ni el consumo crónico de alcohol produjo diferencias en la enzima (Figura 5).

\section{Discusión}

El hallazgo que tratamiento con fenofibrato aumenta no solo la actividad de la catalasa (Karahanian et al., 2014; Rivera-Meza et al., 2017) sino también los niveles hepáticos de ADH1 es valioso para entender los efectos extraordinarios de este fármaco en aumentar los niveles de acetaldehído en sangre y, por último, disminuir el consumo voluntario de alcohol en ratas. Dicho efecto de la administración de fibratos en la expresión de ADH1 en el hígado fue descrito anteriormente por Kramer et al. (2003), que informaron un aumento en la transcripción de este gen en ratas tratadas con clofibrato. Más recientemente, Ferguson, Most, Blednov y Harris (2014) informaron de aumentos en los niveles de la transcripción de ADH1 en el hígado después de un tratamiento de ocho días con fenofibrato o tesaglitazar (un agonista dual PPAR $\alpha$ y PAR $\gamma$ ), pero no bezafibrato (un agonista PAN-PPAR).

Aunque los estudios pioneros de Lieber (1988) y otros (Vidal, Perez, Morancho, Pinto y Richart, 1990) mostraron que el consumo de alcohol no induce el ADH hepático, también hay estudios que muestran que la administración intragástrica de $13 \mathrm{~g} / \mathrm{kg} /$ día de etanol a ratas aumentó los niveles de mRNA (Badger et al., 2000; Deaciuc, Arteel, Peng, Hill y McClain, 2004), proteína y actividad enzimática (He, Ronis y Badger, 2002) de ADH1. De manera similar, también encontramos que el consumo crónico de etanol a $\sim 7 \mathrm{~g} / \mathrm{kg}$ /día per se aumentó los niveles hepáticos de ADH1 en ratas. Puede haber un efecto dependiente de la dosis de etanol, dado que He et al. (2002) informaron un aumento de 3,3 veces de los niveles de ADH1 con $13 \mathrm{~g} / \mathrm{kg} /$ día de etanol, mientras que en nuestro estudio detectamos un aumento de 1,6 veces con $\sim 7 \mathrm{~g} / \mathrm{kg} /$ día. Es de interés que el tratamiento simultáneo con etanol y fenofibrato produjo los niveles más altos de ADH1, lo que sugiere el efecto aditivo entre ambos tratamientos. Una explicación posible de la expresión aumentada de $\mathrm{ADH} 1$ por el tratamiento con fenofibrato podría ser la presencia de un elemento de respuesta al PPAR (PPRE) en el promotor del gen ADH1 de rata. Buscamos una secuencia de consenso PPRE (Tzeng et al., 2015) en este promotor, pero no hallamos presencia de alguno. Entonces, es posible que el PPAR $\alpha$ estimula la expresión de ADH1 a través de un mecanismo indirecto.

De manera diferente a los resultados de otros estudios, en los que los niveles de ALDH2 mRNA en ratones disminuyeron debido a clofibrato (Moffit et al., 2007) o tesaglitazar (Ferguson et al., 2014), no observamos cambio alguno en los niveles proteicos de ALDH2 producidos por fenofibrato en nuestro modelo de ratas con consumo crónico de alcohol. Este último resultado es de interés para conseguir aumentar los niveles de acetaldehído en sangre. Crabb et al. (2001) informaron una caída ligera en la expresión de ALDH2 en el hígado de ratas tratadas con clofibrato y en ratones tratados con WY14643 (un agonista PPAR $\alpha$ ); no obstante, WY14643 tuvo el mismo efecto en ratones knockout para PPAR $\alpha$, lo que sugiere que dicho efecto no sería mediado por PPAR $\alpha$.

Las mediciones de la actividad enzimática de ADH1 y ALDH2 en el hígado mostraron resultados similares a la cuantificación de proteínas mediante Western blot: ADH1 mostró el nivel más alto de actividad en los animales bebedores de alcohol tratados con fenofibrato, con un aumento menor en los animales tratados solo con fenofibrato. Aunque en un estudio anterior (Karahanian et al., 2014) informamos que las dosis de fenofibrato $50 \mathrm{mg} / \mathrm{kg} /$ día no alteraron la actividad de ADH1 en el hígado, en el estudio actual medimos las enzimas en ratas tratadas con fenofibrato $100 \mathrm{mg} / \mathrm{kg} /$ día, y sugerimos que puede ser necesaria esta dosis más elevada de fenofibrato para aumentar la actividad de ADH1. Se ha demostrado que una actividad más elevada de $\mathrm{ADH} 1$ en el hígado está relacionada, de forma directa, con una mayor producción de acetaldehído cuando se consume etanol, en ambos modelos: con animales (Rivera-Meza et al., 2010; Rivera-Meza, Quintanilla y Tampier, 2012) y con humanos (Lee, Höög y Yin, 2004). Se ha identificado que los polimorfismos genéticos en humanos producen una actividad cuarenta veces mayor de $\mathrm{ADH}$ (ADH1B2) que el alelo normal (Lee et al., 2004). De forma interesante, este alelo es relativamente común entre personas de origen asiático, donde los portadores de esta mutación han mostrado tener un efecto protector contra el alcoholismo (Thomasson et al., 1991; Thomasson et al., 1994). Ser portador de una ADH "más rápida” resultaría en una acumulación rápida inicial de acetaldehído (Quintanilla, Tampier, Sapag, Gerdtzen y Israel, 2007) cuando estas personas consumen alcohol, lo que causaría los efectos adversos descritos anteriormente. Los datos aportados y objeto de discusión apoyan la idea que el efecto de fenofibrato en aumentar la actividad de ADH1 tendría un papel importante (además de la catalasa) en explicar la efectividad de este fármaco para reducir el consumo de alcohol en ratas.

Aunque también es verdad que el aumento de los niveles de acetaldehído en sangre explican varios de los efectos 
secundarios de disulfiram, este medicamento per se tiene otras características que explican su toxicidad. El disulfiram es una droga no específica que también inhibe la conversión de dopamina a noradrenalina, y el agotamiento de noradrenalina en el sistema cardiovascular potencia la acción del acetaldehído sobre tejidos miocardios y vasculares para provocar rubor, taquicardia e hipotensión (Sinclair, Chambers, Shiles y Baldwin, 2016). Además, el disulfiram inhibe el $\mathrm{N}$-acetiltransferasa y varios miembros de la familia de los citocromos y, por tanto, aumenta la toxicidad de una variedad de otras drogas (Frye y Branch, 2002). Otra desventaja de este medicamento es la marcada variabilidad entre sujetos en los niveles en plasma de disulfiram y sus metabolitos (Faiman, Jensen y Lacoursiere, 1984) y, debido a su vía metabólica compleja, la gran variabilidad individual en la respuesta (Mays et al., 1995).

Como indicamos anteriormente, el disulfiram carece de efectividad para reducir el consumo de etanol en un porcentaje considerable de pacientes (Christensen et al., 1991; Skinner et al., 2014; Yoshimura et al., 2014). Proponemos una explicación para la falta de respuesta al disulfiram de algunos individuos: aunque es ampliamente reconocido que un aumento de los niveles periféricos de acetaldehído genera afectos aversivos al etanol, una idea que ha cobrado fuerza es que el acetaldehído a nivel central, cuando es generado directamente en el cerebro a partir de etanol, tiene propiedades de refuerzo hacia el consumo de alcohol (Israel et al., 2013; Israel et al., 2015). A diferencia del hígado, la ADH1 no se expresa en el cerebro; en su lugar, la catalasa es la principal enzima responsable de oxidar etanol a acetaldehído. Después, como ocurre en el hígado, la ALDH2 se encarga de eliminar el acetaldehído acumulado. En un estudio anterior, informamos que al inhibir la expresión de la catalasa en el área tegmental ventral del cerebro (implicado en la liberación de dopamina en el sistema límbico de recompensa) y, de esta manera, bloquear la producción de acetaldehído en esta región, se inhibe la capacidad del etanol para generar adicción en las ratas UChB (Karahanian et al., 2011). De manera similar, el mismo efecto ocurre al aumentar la eliminación de acetaldehído mediante la sobreexpresión de ALDH2 (Karahanian et al., 2015). Consideramos que una de las desventajas del disulfiram es que puede cruzar la barrera hematoencefálica (Hellström y Tottmar, 1982) e inhibir la actividad cerebral de ALDH2. Por esto, cuando una persona consume alcohol, la catalasa genera acetaldehído en el cerebro que se queda sin metabolizar y, como mencionamos, podría generar un efecto de refuerzo. Por esto, nuestra hipótesis es que la aversión generada por acetaldehído periférico podría ser rebasada por los efectos de refuerzo a nivel central. Por tanto, para hallar una droga más efectiva que el disulfiram para reducir el consumo de alcohol, dicha droga, en condiciones ideales, estimularía la producción de acetaldehído periférica en lugar de ce- rebral. Opinamos que el fenofibrato cumpliría con estas características perfectamente, dado que hemos mostrado que no aumenta la actividad cerebral de la catalasa.

En general, estos estudios muestran que el tratamiento con fenofibrato no solo aumenta la actividad hepática de la catalasa en ratas bebedoras de alcohol, como indicamos previamente (Karahanian et al., 2014; Rivera-Meza et al., 2017), sino también los niveles de actividad enzimática de ADH1, mientras que ALDH2 se mantuvo igual. Estos resultados son valiosos para entender por qué el fenofibrato tiene efectos extraordinarios en aumentar los niveles de acetaldehído en sangre de animales bebedores de alcohol. No obstante, son necesarios más estudios para demostrar la eficacia del fenofibrato para reducir el consumo de alcohol (e.g., para estudiar los efectos posibles a nivel central que disminuirían la motivación de beber, más allá de la aversión generada por el acetaldehído periférico).

\section{Reconocimientos}

Este estudio recibió apoyo de FONDECYT \#1150850 y CONICYT Anillo ACT1411

\section{Conflicto de intereses}

Los autores declaran la inexistencia de conflicto de intereses.

\section{Referencias}

Badger, T. M., Hoog, J. O, Svensson, S., McGehee, R. E. Jr., Fang, C., Ronis, M. J. y Ingelman-Sundberg M. (2000). Cyclic expression of class I alcohol dehydrogenase in male rats treated with ethanol. Biochemical and Biophysical Research Communications, 274, 684-688. doi:10.1006/ bbrc.2000.3186.

Blednov, Y. A., Black, M., Benavidez, J. M., Stamatakis, E. E. y Harris, R.A. (2016). PPAR agonists: I. Role of receptor subunits in alcohol consumption in male and female mice. Alcoholism: Clinical and Experimental Research, 40, 553-562. doi:10.1111/acer.12976.

Christensen, J. K., Moller, I. W., Ronsted, P., Angelo, H. R. y Johansson, B. (1991). Dose-effect relationship of disulfiram in human volunteers. I: Clinical studies. Pharmacology EF Toxicology, 68, 163-165. doi:10.1111/j.1600-0773.1991. tb01215.x.

Crabb, D. W., Pinaire, J., Chou, W.Y., Sissom, S., Peters, J.M., Harris, R. A. y Stewart, M. (2001). Peroxisome proliferator-activated receptors (PPAR) and the mitochondrial aldehyde dehydrogenase (ALDH2) promoter in vitro and in vivo. Alcoholism Clinical and Experimental Research, 25, 945-952. doi:10.1111/j.1530-0277.2001.tb02301.x.

Deaciuc, I. V., Arteel, G. E., Peng, X., Hill, D. B. y McClain, C. J. (2004). Gene expression in the liver of rats fed alco- 
hol by means of intragastric infusion. Alcohol, 33, 17-30. doi:10.1016/j.alcohol.2004.04.001.

Dupuy, O., Flocard, F., Vial, C., Rode, G., Carles, N., Boisson, D. y Flechaire, A. (1995). Disulfiram toxicity. Le Revue de Medecine Interne, 16, 67-72. doi:10.1016/02488663(96)80667-9.

Faiman, M. D., Jensen, J. C. y Lacoursiere, R. B. (1984). Elimination kinetics of disulfiram in alcoholics after single and repeated doses. Clinical Pharmacology and Therapeutics, 36, 520-526. doi:10.1038/clpt.1984.213.

Ferguson, L. B., Most, D., Blednov, Y. A. y Harris, R. A. (2014). PPAR agonists regulate brain gene expression: relationship to their effects on ethanol consumption. Neuropharmacology, 86, 397-407. doi:10.1016/j.neuropharm.2014.06.024.

Frye, R. F. y Branch, R. A. (2002). Effect of chronic disulfiram administration on the activities of CYP1A2, CYP2C19, CYP2D6, CYP2E1, and N-acetyltransferase in healthy human subjects. British Journal of Clinical Pharmacology, 53, 155-162. doi:10.1046/j.1365-2125.2002.01522.x.

Gervois, P., Fruchart, J. C. y Staels, B. (2007). Drug Insight: mechanisms of action and therapeutic applications for agonists of peroxisome proliferator-activated receptors. Nature Clinical Practice Endocrinology E Metabolism, 3, 145156. doi:10.1038/ncpendmet0397.

Haile, C. N. y Kosten, T. A. (2017). The peroxisome proliferator-activated receptor alpha agonist fenofibrate attenuates alcohol self-administration in rats. Neuropharmacology, 116, 364-370. doi:10.1016/j.neuropharm.2017.01.007.

Handler, J. A. y Thurman, R. G. (1988a). Catalase-dependent ethanol oxidation in perfused rat liver. Requirement for fatty-acid-stimulated $\mathrm{H} 2 \mathrm{O} 2$ production by peroxisomes. European Journal of Biochemistry, 176, 477-484. doi:10.1111/j.1432-1033.1988.tb14305.x.

Handler, J. A. y Thurman, R. G. (1988b). Hepatic ethanol metabolism is mediated predominantly by catalase-H2O2 in the fasted state. FEBS Letters, 238, 139-141. doi:10.1016/0014-5793(88)80243-6.

He, L., Ronis, M. J. y Badger, T. M. (2002). Ethanol induction of class I alcohol dehydrogenase expression in the rat occurs through alterations in CCAAT/enhancer binding proteins beta and gamma. The Journal of Biological Chemistry, 277, 43572-43577. doi:10.1074/jbc. M204535200.

Hellström, E. y Tottmar, O. (1982). Effects of aldehyde dehydrogenase inhibitors on enzymes involved in the metabolism of biogenic aldehydes in rat liver and brain. Biochemical Pharmacology, 31, 3899-3905. doi:10.1016/0006-2952(82)90308-2.

Israel, Y., Rivera-Meza, M., Karahanian, E., Quintanilla, M. E., Tampier, L., Morales, P. y Herrera-Marschitz, M. (2013). Gene specific modifications unravel ethanol and acetaldehyde actions. Frontiers in Behavioral Neuroscience, 7, 80. doi:10.3389/fnbeh.2013.00080.
Israel, Y., Quintanilla, M. E., Karahanian, E., Rivera-Meza, M. y Herrera-Marschitz, M. (2015). The "first hit" toward alcohol reinforcement: role of ethanol metabolites. Alcoholism Clinical and Experimental Research, 39, 776786. doi:10.1111/acer.12709.

Karahanian, E., Ocaranza, P. y Israel, Y. (2005). Aldehyde dehydrogenase (ALDH2) activity in hepatoma cells is reduced by an adenoviral vector coding for an ALDH2 antisense mRNA. Alcoholism Clinical and Experimental Research, 29, 1384-1389. doi:10.1097/01. alc.0000174909.91034.7c.

Karahanian, E., Quintanilla, M. E., Tampier, L., Rivera-Meza, M., Bustamante, D., Gonzalez-Lira, V., ... Israel, Y. (2011). Ethanol as a prodrug: brain metabolism of ethanol mediates its reinforcing effects. Alcoholism Clinical and Experimental Research, 35, 606-612. doi:10.1111/ j.1530-0277.2011.01439.x.

Karahanian, E., Quintanilla, M. E., Fernandez, K. y Israel, Y. (2014). Fenofibrate -a lipid-lowering drug- reduces voluntary alcohol drinking in rats. Alcohol, 48, 665-670. doi:10.1016/j.alcohol.2014.08.004.

Karahanian, E., Rivera-Meza, M., Tampier, L., Quintanilla, M. E., Herrera-Marschitz, M. y Israel, Y. (2015). Longterm inhibition of ethanol intake by the administration of an aldehyde dehydrogenase-2 (ALDH2)-coding lentiviral vector into the ventral tegmental area of rats. Addiction Biology, 20, 336-344. doi:10.1111/adb.12130.

Kramer, J. A., Blomme, E. A., Bunch, R. T., Davila, J. C., Jackson, C. J., Jones, P. F., ... Curtiss, S. W. (2003). Transcription profiling distinguishes dose-dependent effects in the livers of rats treated with clofibrate. Toxicologic Pathology, 31, 417-431. doi:10.1080/01926230390202353.

Lee, S. L., Höög, J. O. y Yin, S. J. (2004). Functionality of allelic variations in human alcohol dehydrogenase gene family: assessment of a functional window for protection against alcoholism. Pharmacogenetics, 14, 725-732.

Lieber, C. S. (1988). Pathophysiology of alcoholic liver disease. Molecular Aspects of Medicine, 10, 107-146. doi:10.1016/0098-2997(88)90018-0.

Maisel, N. C., Blodgett, J. C., Wilbourne, P. L., Humphreys, K. y Finney, J. W. (2013). Meta-analysis of naltrexone and acamprosate for treating alcohol use disorders: when are these medications most helpful? Addiction, 108, 27593. doi:10.1111/j.1360-0443.2012.04054.x.

Mark, T. L., Kranzler, H. R., Song, X., Bransberger, P., Poole, V. H. y Crosse, S. (2003). Physicians' opinions about medications to treat alcoholism. Addiction, 98, 617-626. doi:10.1046/j.1360-0443.2003.00377.x.

Mays, D. C, Nelson, A. N., Fauq, A. H., Shriver, Z. H., Veverka, K. A., Naylor, S. y Lipsky, J. J. (1995). S-methyl $\mathrm{N}, \mathrm{N}$-diethylthiocarbamate sulfone, a potential metabolite of disulfiram and potent inhibitor of low Km mitochondrial aldehyde dehydrogenase. Biochemical Pharmacology, 49, 693-700. doi:10.1016/0006-2952(94)00504-F. 
Moffit, J. S., Koza-Taylor, P. H., Holland, R. D., Thibodeau, M. S., Beger, R. D., Lawton, M. P. y Manautou, J. E. (2007). Differential gene expression in mouse liver associated with the hepatoprotective effect of clofibrate. Toxicology and Applied Pharmacology, 222, 169-179. doi:10.1016/j.taap.2007.04.008.

Quintanilla, M. E., Israel, Y., Sapag, A. y Tampier, L. (2006). The UChA and UChB rat lines: metabolic and genetic differences influencing ethanol intake. Addiction Biology, 11, 310-323. doi:10.1111/j.1369-1600.2006.00030.x.

Quintanilla, M. E., Tampier, L., Sapag, A., Gerdtzen, Z. y Israel, Y. (2007). Sex differences, alcohol dehydrogenase, acetaldehyde burst, and aversion to ethanol in the rat: a systems perspective. American Journal of Physiology. Endocrinology and Metabolism, 293, E531-537. doi:10.1152/ ajpendo.00187.2007.

Rivera-Meza, M., Quintanilla, M. E., Tampier, L., Mura, C. V., Sapag, A. y Israel, Y. (2010). Mechanism of protection against alcoholism by an alcohol dehydrogenase polymorphism: development of an animal model. FASEB Journal, 24, 266-274. doi:10.1096/fj.09-132563.

Rivera-Meza, M., Quintanilla, M. E. y Tampier, L. (2012). Reduction of ethanol consumption in alcohol-preferring rats by dual expression gene transfer. Alcohol and Alcoholism, 47, 102-108. doi:10.1093/alcalc/agr161.

Rivera-Meza, M., Muñoz, D., Jerez, E., Quintanilla, M. E., Salinas-Luypaert, C., Fernandez, K. y Karahanian, E. (2017). Fenofibrate administration reduces alcohol and saccharin intake in rats: possible effects at peripheral and central levels. Frontiers in Behavioral Neuroscience, 11, 133. doi:10.3389/fnbeh.2017.00133.

Sinclair, J. M. A., Chambers, S. E., Shiles, C. J. y Baldwin, D. S. (2016). Safety and tolerability of pharmacological treatment of alcohol dependence: comprehensive review of evidence. Drug Safety, 39, 627-645. doi:10.1007/ s40264-016-0416-y.

Skinner, M. D., Lahmek, P., Pham, H. y Aubin, H. J. (2014). Disulfiram efficacy in the treatment of alcohol dependence: a meta-analysis. PLoS One 9, e87366. doi:10.1371/ journal.pone.0087366.

Thomasson, H. R., Edenberg, H. J., Crabb, D. W., Mai, X. L., Jerome, R. E., Li, T.K., ... Yin, S. J. (1991). Alcohol and aldehyde dehydrogenase genotypes and alcoholism in Chinese men. American Journal of Human Genetics, 48, 677-681.

Thomasson, H. R., Crabb, D. W., Edenberg, H. J., Li, T. K., Hwu, H. G., Chen, C. C., ... Yin, S. J. (1994). Low frequency of the $\mathrm{ADH} 2 * 2$ allele among Atayal natives of Taiwan with alcohol use disorders. Alcoholism Clinical and Experimental Research, 18, 640-643. doi:10.1111/j.1530-0277.1994.tb00923.x.

Tzeng, J., Byun, J., Park, J. Y., Yamamoto, T., Schesing, K., Tian, B., ... Oka, S. (2015). An ideal PPAR response element bound to and activated by PPAR $\alpha$. PLoS One, 10, e0134996. doi:10.1371/journal.pone.0134996.

Vidal, F., Perez, J., Morancho, J., Pinto, B. y Richart C. (1990). Hepatic alcohol dehydrogenase activity in alcoholic subjects with and without liver disease. Gut, 31, 707-711. doi:10.1136/gut.31.6.707.

Yoshimura, A., Kimura, M., Nakayama, H., Matsui, T., Okudaira, F., Akazawa, S., ... Higuchi, S. (2014). Efficacy of disulfiram for the treatment of alcohol dependence assessed with a multicenter randomized controlled trial. Alcoholism Clinical and Experimental Research, 38, 572-578. doi:10.1111/acer.12278. 